\title{
ON PRIMAL IDEALS
}

\section{LADISLAS FUCHS}

1. Introduction. In my paper On quasi-primary ideals ${ }^{1}$ I have added a fifth decomposition of an ideal to those given by E. Noether. ${ }^{2}$ Thus in a commutative ring with unit element in which the maximal condition is satisfied there are in general five distinct decompositions of an ideal; namely as shortest ${ }^{3}$ intersection of a finite set of (i) irreducible, (ii) primary, (iii) quasi-primary, (iv) relatively-prime-indecomposable, (v) direct-indecomposable ideals. The components in (iv) and in (v) are unique, while in (i), (ii), (iii) the associated prime ideals, but not necessarily the components themselves, are unique. In (i) one prime ideal may belong to more than one component, but always to the same number of components. Each prime ideal that occurs in (i) occurs even in (ii) but with a single multiplicity, while in (iii) only the minimal prime ideals of (ii) occur, each but once.

The comparison of the features of the prime ideals associated with components of (ii) and of (iii) suggests that, perhaps, a new type of decomposition may be defined, one whose components are associated with just the maximal prime ideals of (ii). The sought type of ideals will be realised by those which coincide with their single "principal component" in the sense of W. Krull. ${ }^{4}$ These ideals will be defined here more directly, again by making use of the familiar concept of "relative primeness." However, it must be emphasized that the method used here is different from Krull's method. Moreover it seems quite questionable whether all principal components are primal.

2. Definition and preliminaries. We shall say that $g$ is a primal ideal if the elements which are not prime ${ }^{b}$ to it form an ideal $\mathfrak{p}$ called

Received by the editors June 15, 1948 and, in revised form, August 15, 1948.

1 Acta Univ. Szeged. vol. 11 (1947) pp. 174-183. An ideal is quasi-primary if no product of two elements belongs to it unless a power of one of them belongs to it, or, otherwise, if its radical is prime.

2 E. Noether, Idealtheorie in Ringbereichen, Math. Ann. vol. 83 (1921) pp. 24-66.

3 The term "shortest" means that no component may simply be omitted and no intersection of more than one component is again of the same type.

4W. Krull, Idealtheorie in Ringen ohne Endlichkeitsbedingung, Math. Ann. vol. 101 (1929) pp. 729-744. If $\mathfrak{p}$ is a maximal prime divisor of $\mathfrak{a}$ (that is, $\mathfrak{p}$ is a maximal ideal that contains no element prime to $a)$, then the principal component $a(p)$ is defined to consist of all elements whose product by a properly chosen element not in $p$ belongs to $a$.

b $a$ is called (relatively) prime to $\mathfrak{b}$, if $a b \in \mathfrak{b}$ is impossible unless $b \in \mathfrak{b} ;$ cf. Noether, loc. cit. footnote 2 , p. 45 . 
the adjoint ideal of $\mathfrak{g}$. As $b c$ together with $b$ is not prime to $\mathfrak{g}$ whatever the element $c$, what we require is that together with two elements their difference shall be non-prime to $\mathrm{g}$.

Evidently, $\mathfrak{g}$ is a primal ideal and $\mathfrak{p}$ is adjoint to $\mathfrak{g}$ if and only if $a b \in \mathfrak{g}$ and $b \in_{\mathfrak{g}}$ imply $a \in \mathfrak{p}$ and, conversely, whenever $a \in \mathfrak{p}$, there always exists an element $b$ not in $\mathfrak{g}$ such that $a b \in \mathfrak{g}$.

The elements non-prime to $g$ represent in the residue class ring $\Re / g$ the zero factors, therefore the definition of primal ideals may also be formulated as follows: in $\Re / g$ the zero factors form an ideal, namely $\mathfrak{p} / \mathfrak{g}$.

The product of two elements prime to an ideal $g$ is again prime to it, ${ }^{6}$ since, assuming $b$ and $c$ prime to $\mathfrak{g}, a b c \in \mathfrak{g}$ implies $a b \in \mathfrak{g}$ and this implies $a \in \mathfrak{g}$. Therefore no product of two elements not belonging to $\mathfrak{p}$ belongs to $\mathfrak{p}$, thus the adjoint ideal is prime. This fact shows the analogy between the ideals adjoint to primal ideals and those associated with quasi-primary ideals.

The primal ideals may be considered as an extension of the idea of primary ideals. Indeed, the elements non-prime to a primary ideal are just the elements of its prime radical. But there are in general primal ideals which are not primary; moreover a primal ideal may be quasi-primary and need not be primary. To illustrate this, let us consider the ring of polynomials in $x$ and $y$ with rational numbers for their coefficients. In this ring $\mathfrak{x}=\left(x^{2}, x y\right)$ is a quasi-primary ideal with the radical $(x)$ and is primal with $(x, y)$ as adjoint prime ideal. $\mathfrak{x}$ is not primary for $x y \in \mathfrak{x}$ and neither $x$ nor any power of $y$ belongs to $\mathfrak{x}$. As another illustration we may refer to a valuation ring with $\dot{a}$ non-archimedian value-group, ${ }^{7}$ where all ideals are both quasiprimary [for if $b c \in \mathfrak{a}$, then either $b^{2} \in \mathfrak{a}$ or $c^{2} \in \mathfrak{a}$ according as $c$ divides $b$ or $b$ divides $c$ ] and primal [for if $b_{1} c_{1} \in \mathfrak{a}, b_{2} c_{2} \in \mathfrak{a}$ with $c_{1} \notin \mathfrak{a}, c_{2} \notin \mathfrak{a}$ and, for example, $c_{1}$ divides $c_{2}$, then $\left.c_{2}\left(b_{1}-b_{2}\right) \in \mathfrak{a}\right]$, but not necessarily primary!

If, however, an ideal $g$ is quasi-primary and primal, and at the same time its prime radical and its adjoint prime ideal coincide, then $\mathfrak{g}$ is necessarily primary; for $a b \in g, b \notin g$ imply that $a$ belongs to the adjoint ideal, that is, to the radical, therefore it follows that a certain power of $a$ belongs to $\mathfrak{g}: \mathfrak{g}$ is indeed primary. ${ }^{\mathfrak{g}}$

One simple remark will be inserted here in order to make the con-

\footnotetext{
- With the terminology of Krull: the system of elements prime to $g$ is multiplicatively closed.

' W. Krull, Allgemeine Bewertungstheorie, J. Reine Angew. Math. vol. 167 (1932) pp. $160-196$.

- Cf. Krull's corollary to Satz 6, loc. cit. footnote 4, p. 737.
} 
nection between primal ideals and principal components more apparent. Namely, we shall show that the principal component $\mathfrak{a}(\mathfrak{p})$ of $\mathfrak{a}$ belonging to the maximal prime ideal $\mathfrak{p}$ is a multiple of the intersection $\mathfrak{a}^{*}(\mathfrak{p})$ of all primal divisors of $\mathfrak{a}$ whose adjoint prime ideal is contained in $\mathfrak{p}$. Indeed, if $\mathfrak{g}$ is such a primal ideal and $a \in \mathfrak{a}(\mathfrak{p})$, then by definition there is an element $b$ not in $\mathfrak{p}$ such that $a b \in \mathfrak{a}$. Now $a b \in \mathfrak{g}$, and this implies $a \in \mathfrak{g}$, for $b \notin \mathfrak{p}$ must be prime to $\mathfrak{g}$; therefore $\mathfrak{a}(\mathfrak{p}) \subseteq \mathfrak{g}$. It is, however, an open question whether $\mathfrak{a}^{*}(\mathfrak{p})$ is always equal to $\mathfrak{a}(\mathfrak{p})$ or not.

The great importance of primal ideals lies in the fact that the following theorem may be proved in complete generality, without making use of any condition.

ThEOREM 1. Every irreducible ideal is primal.

The proof is based on a simple law on forming ideal quotients. ${ }^{9}$ Let $i$ be any irreducible ideal and $b_{1}, b_{2}$ two elements which are not prime to $\mathfrak{i}$. Then $\mathfrak{i}:\left(b_{1}\right)$ and $\mathfrak{i}:\left(b_{2}\right)$ are proper divisors of $\mathfrak{i}$, hence their intersection $\mathfrak{i}:\left(b_{1}\right) \cap \mathfrak{i}:\left(b_{2}\right)=\mathfrak{i}:\left(\left(b_{1}\right)+\left(b_{2}\right)\right)$ cannot equal $\mathfrak{i}$, consequently, the element $b_{1}-b_{2}$ cannot be prime to $i$, q.e.d.

3. The intersection of primal ideals. We now inquire when the intersection of primal ideals is again primal. It will seem no doubt somewhat surprising at first glance that the intersection of two primal ideals with the same adjoint prime ideal is not necessarily primal, not even in rings with basis theorem. For instance, in the polynomial domain considered above $\left(x^{2}, x y\right)$ and $\left(x y, y^{2}\right)$ are primal ideals both adjoint to $(x, y)$, but they have for intersection a nonprimal ideal, namely $(x y) .{ }^{10}$ But if we restrict ourselves merely to reduced ${ }^{11}$ intersections, we may state the following theorem.

THEOREM 2. The reduced intersection of a finite number of primal ideals $\mathfrak{a}=\mathfrak{g}_{1} \cap \cdots \cap \mathfrak{g}_{n}$ with with $\mathfrak{p}_{1}, \cdots, \mathfrak{p}_{n}$ as adjoint prime ideals is again primal if and only if one prime ideal $\mathfrak{p}_{j}$ divides all other $\mathfrak{p}_{i}$. Then $\mathfrak{p}_{j}$ is adjoint to a.

From the hypothesis that the intersection $\mathfrak{g}_{1} \cap \ldots \cap \mathfrak{g}_{n}$ is reduced, we infer by a theorem of $E$. Noether ${ }^{12}$ that $b$ is not prime to $a$ if and only if it is not prime to at least one of $\mathfrak{g}_{i}$, that is, if and only if

o $\mathfrak{a}:\left(\mathfrak{b}_{1}+\mathfrak{b}_{2}\right)=\mathfrak{a}: \mathfrak{b}_{1} \cap a b_{2}$. See, for example, B. L. van der Waerden, Moderne Algebra, vol. 2, 2d ed., Berlin, 1940, p. 25.

${ }^{10} x$ and $y$ are not prime to $(x y)$, but $x-y$ is prime to it.

11 "Reduced" means that no component may be replaced by one of its proper divisors.

12 See Noether, loc. cit. footnote 2, Satz X, p. 45. 
$b$ belongs to at least one of the $p_{i}$. This tells us that $a$ is primal if and only if the union of the elements of all $\mathfrak{p}_{i}$ is a prime ideal. Since the sufficiency of the stated condition is thus clear, to bring the proof to an end we must still show that the union of the elements of a finite set of primes is not a prime ideal again unless one divides all others.

Let $\mathfrak{p}_{1}^{*}, \cdots, \mathfrak{p}_{k}^{*}$ denote the different maximal ones of $\mathfrak{p}_{1}, \cdots, \mathfrak{p}_{n}$, that is, those which are divided by no different one in the set of $\mathfrak{p}_{1}, \cdots, \mathfrak{p}_{n}$; further assume $k>1$. None of $\mathfrak{p}_{l}{ }^{*}$ divides another one; we can therefore find elements $p_{l}(l=1, \cdots, k)$ contained in $\mathfrak{p}_{1}^{*} \cap \cdots \cap \mathfrak{p}_{l-1}^{*} \cap \mathfrak{p}_{l+1}^{*} \cap \cdots \cap \mathfrak{p}_{k}^{*}$ but not in $\mathfrak{p}_{l}^{*}$. If a were primal, the fact that the $p_{l}$ are not prime to a would imply that $p=p_{1}+\ldots$ $+p_{k}$ is not prime to $a$. Hence $p$ would belong to one of the $p_{i}$ and so, a fortiori, to one of $p_{l}{ }^{*}$. This is, however, a contradiction, since each $p_{m}$ except $p_{l}$ belongs to $p_{l}^{*}$. The proof is thus completed.

As an immediate consequence of Theorem 2 we may establish that in a ring with maximal condition an ideal is primal if and only if one of the prime ideals associated with its representation (ii) contains all the others. The assertion becomes evident in view of Theorem 2 if one takes into account that the same prime ideals are associated with representation (i) as with (ii) and, further, that a shortest irreducible representation is at the same time reduced. ${ }^{13}$ This fact implies that if $\mathfrak{p}$ adjoint to the primal ideal $\mathfrak{g}$ is a minimal prime ideal in a ring with maximal condition, then $g$ is primary, for its associated prime ideals are multiples of $p$ and so necessarily equal to $\mathfrak{p} .^{14}$

4. The existence of primal decompositions. Now we shall be concerned with decompositions into primal ideals. The bare existence of primal decompositions is all that we can prove in general.

TheoRem 3. Every ideal is representable as the intersection of its primal divisors.

Since the unit ideal $\boldsymbol{D}$ is primal and divides each ideal, the intersection of the primal divisors of an arbitrary ideal $\mathfrak{a}$ exists and, what is quite evident, divides a. What we have thus to prove is that if $a$ does not belong to $\mathfrak{a}$, there is a primal divisor of $\mathfrak{a}$ which does not contain $a$. By Zorn's well known lemma ${ }^{15}$ we may conclude that there exists an ideal $g$ dividing $\mathfrak{a}$ but not containing $a$ such that each proper

13 Cf. Noether, loc. cit. footnote 2, Hilfssatz II, p. 35.

14 The intersection of a finite number of primary ideals associated with the same prime ideal is primary again; see, for example, van der Waerden, loc. cit. footnote $9, \mathrm{p}$. 32.

${ }^{15} \mathrm{M}$. Zorn, A remark on method in transfinite algebra, Bull. Amer. Math. Soc. vol. 41 (1935) pp. 667-670. 
divisor of $g$ contains $a$. Let $b_{1}, b_{2}$ be elements not prime to $\mathfrak{g}$ and determine $c_{1}, c_{2}$ not in $\mathfrak{g}$ such that $b_{1} c_{1} \in \mathfrak{g}, b_{2} c_{2} \in \mathfrak{g}$. The ideals $\mathfrak{g}+\left(c_{1}\right)$ and $\mathfrak{g}+\left(c_{2}\right)$ are proper divisors of $\mathfrak{g}$, therefore both contain $a, a \in \mathfrak{g}$ $+\left(c_{1}\right)$ and $a \in \mathfrak{g}+\left(c_{2}\right)$. We hence have $a b_{1} \in \mathfrak{g} b_{1}+\left(b_{1} c_{1}\right) \subset g$ and similarly $a b_{2} \in \mathfrak{g}$, so that one may see that $b_{1}-b_{2}$ is not prime to $g$, for, while $a\left(b_{1}-b_{2}\right) \in \mathfrak{g}, a$ itself does not belong to $\mathfrak{g} .^{16}$

5. Finite decompositions. In order to analyze the finite primal decompositions, instead of confining ourselves merely to rings with ascending chain condition we shall consider somewhat more general rings. We want to establish, as far as we can, the existence of a decomposition into a finite set of primal ideals and even the unicity of the prime ideals adjoint to the components. It appears to be necessary to restrict ourselves to rings in which every ideal is representable as the intersection of a finite number of irreducible ideals as well as to normal representations. ${ }^{17}$ Our stated problem is completely solved only in such rings and for such decompositions.

Given any ideal $a$, we start with one of its finite shortest, and so necessarily reduced, irreducible representations and unite all components whose adjoint prime ideal $p$ is the same as or is a multiple of an arbitrary maximal $\mathfrak{p}_{j}$, say, of $\mathfrak{p}_{1}$. Then, by Theorem 2 , we obtain a new primal ideal with $p_{1}$ as adjoint prime ideal. Thus proceeding, we finally arrive at a shortest primal decomposition which will be reduced $^{18}$ and hence normal.

THEOREM 4. In a ring where each ideal may be represented as the intersection of a finite set of irreducible ideals, each ideal possesses a normal decomposition into a finite number of primal ideals.

6. The unicity statement. Finally, we establish the unicity of the

16 I am indebted to Professor Nathan Jacobson for having called my attention to N. H. McCoy's paper, Subdirectly irreducible commutative rings, Duke Math. J. vol. 12 (1945) pp. 381-387, whose Theorem 1 includes Theorem 3 of the present paper. The residue class ring $\Re / g$ with respect to the ideal $g$ constructed in the proof of Theorem 3 is subdirectly irreducible in the sense of G. Birkhoff (Bull. Amer. Math. Soc. vol. 50 (1944) pp. 761-768); further, $\Re / \mathfrak{a}$ is the subdirect union of the subdirectly irreducible rings $\Re / g$. McCoy's cited theorem (especially part (ii)) asserts that in $\Re / g$ the divisors of zero form an ideal, namely $\mathfrak{p} / \mathfrak{g}$, that is, $g$ is primal. (McCoy's Theorem 2 corresponds to the trivial case where $\mathrm{o}$ is adjoint to $\mathrm{g}$ ).

${ }^{17}$ We use the term "normal" to mean representations which are shortest and reduced.

18 Noether has proved (loc. cit. footnote 2, pp. 36-37) that if all components may be represented as the intersection of a finite number of irreducible ideals, then the reducedness does not alter during the procedure of joining some components into one ideal. 
adjoint prime ideals of normal primal decompositions. ${ }^{19}$

If $\mathfrak{a}=\mathfrak{g}_{1} \cap \ldots \cap \mathfrak{g}_{n}$ and $\mathfrak{a}=\mathfrak{g}_{1}^{*} \cap \ldots \cap \mathfrak{g}_{m}^{*}$ are two normal primal decompositions with the adjoint prime ideals $\mathfrak{p}_{1}, \cdots, \mathfrak{p}_{n}$ and $p_{1}^{*}, \cdots, p_{m}^{*}$ respectively, we first show that each $p_{i}(i=1, \cdots, n)$ is divided by one of $\mathfrak{p}_{j}^{*}(j=1, \cdots, m)$ and each $\mathfrak{p}_{j}^{*}$ by one of $\mathfrak{p}_{i}$. For if $p_{1}$ were divided by no one of $p_{j}^{*}$, we should get a contradiction as follows. The hypothesis admits of choosing an element $p_{j}$ in $p_{1} \cap p_{1}{ }^{*}$ $\cap \cdots \cap p_{j-1}^{*} \cap p_{j+1}^{*} \cap \cdots \cap p_{m}^{*}\left(\right.$ for $j=1, \cdots, m$ ) such that $p_{j}$ does not belong to $p_{j}^{*}$. Then $p=p_{1}+\cdots+p_{m}$ will belong to $p_{1}$ but to none of $\mathfrak{p}_{j}^{*}$, a contradiction, since $p$ is not prime to $\mathfrak{a}$, and therefore, if we use Theorem 2 , it must belong to one of $p_{j}^{*}$.

If now $p_{1}^{*}$ divides $p_{1}$ and is divided by $\mathfrak{p}_{t}$, then $p_{t}$ divides $p_{1}$; this is impossible unless $\mathfrak{p}_{t}=\mathfrak{p}_{1}$. Hence we get $p_{1}=p_{1}^{*}$ and the theorem that we are going to formulate is proved.

TheOREM 5. In two finite normal primal decompositions of an ideal the numbers of the components as well as their adjoint prime ideals are necessarily the same. ${ }^{20}$

7. An illustration. An example will establish that the primal decomposition coincides in general with no other one of (i)-(v). In the polynomial ring of $x, y, z$ decompositions (ii) and (iii) of the ideal $\mathfrak{w}=\left(x^{2} y, x y^{2} z^{2}\right)$ are:

$$
\mathfrak{w}=(x) \cap(y) \cap\left(x^{2}, y^{2}\right) \cap\left(x^{2}, z^{2}\right)
$$

with $(x),(y),(x, y),(x, z)$ as associated prime ideals;

$$
\mathfrak{w}=\left(x^{2}, x y^{2} z^{2}\right) \cap(y)
$$

with the radicals $(x),(y)$.

A normal primal decomposition of $\mathfrak{w}$ is $\mathfrak{w}=\left(x^{2} y, x y^{2}\right) \cap\left(x^{2}, z^{2}\right)$, the adjoint prime ideals being $(x, y)$ and $(x, z)$. It is interesting that in this case there exists a shortest quasi-primary decomposition which is at the same time normal primal, namely $\mathfrak{w}=\left(x^{2}, x z^{2}\right) \cap\left(x^{2} y, y^{2}\right)$ with the radicals $(x),(y)$ and with the adjoint prime ideals $(x, z)$, $(x, y)$.

Finally, $\mathfrak{w}$ is a relatively-prime-indecomposable ideal.

BUdAPEST, HUNGARY

10 Without reducedness the statement is not true. For example, $(x y)=(x) \bigcap(y)$ $=\left(x^{2}, x y\right) \bigcap(y)=(x) \bigcap\left(x y, y^{2}\right)=\left(x^{2}, x y\right) \bigcap\left(x y, y^{2}\right)$ where the adjoint prime ideals are $(x),(y) ;(x, y),(y) ;(x),(x, y) ;(x, y),(x, y)$.

${ }^{20}$ On using the unicity of the associated prime ideals of decomposition (i), Theorem 5 is evident in rings with basis theorem. 